\section{Récepteurs \\ gustatifs \\ des molécules \\ sucrantes \\ et antisucrantes}

receptor 1 ), qui relaient la sensation du goût sucré, sont en fait impliqués dans des systèmes intégrés de chémodétection des produits sucrants circulant dans l'organisme entier. La recherche de leurs rôles dans l'absorption intestinale, la régulation métabolique et l'homéostasie glucidique, notamment, est en plein essor. L'impact biologique, en dehors du système gustatif, des sucrants naturels, des édulcorants mais aussi des molécules antisucrantes moins connues, est l'objet de discussions. La notion de sucrant inverse est introduite et illustrée par la découverte récente que deux classes majeures de composés appartenant respectivement à la médecine (fibrates) et à l'agriculture (phénoxyherbicides), sont en fait des inhibiteurs des récepteurs TlR spécifiques à l'homme. <

La fonction des récepteurs gustatifs TlRl et TlR2 est restée méconnue jusqu'à la découverte de leur partenaire commun, le récepteur TIR3. Le récepteur chémosenseur des produits sucrants est composé des sous-unités TlR2 et TlR3, tandis que le récepteur chémosenseur umami ${ }^{1}$ (le composant principal de cette saveur est le glutamate) est composé des sous-unités TIRl et TIR3 [1-3]. Ces récepteurs font partie de la classe $C$ de la famille des RCPG (récepteurs couplés aux protéines G) [4], avec un large domaine extracellulaire composé d'un module Venus flytrap (VFTM) liant les ligands de type orthostérique et d'une région dite riche en cystéine (CRD) qui lie le domaine VFTM au domaine transmembranaire à 7 hélices qui est caractéristique des RCPG (Figure 1). Des études récentes montrent que le récepteur TlR2/TIR3 a 4 à 6 domaines d'interaction différents avec les ligands [5], ce qui lui permet de répondre à une grande variété de composés sucrants

${ }^{1}$ Parmi les cinq goûts fondamentaux, l'umami fut découvert en 1908 par Kikunae Ikeda qui, en étudiant le goût du dashi à base de kombu (bouillon à base d'algue), révéla que le composant-clé de cette saveur était le glutamate et lui donna le nom d'umami. monelline, thaumatine ${ }^{4}$ ) (Figure 1).

\section{La chémosensation des sucrants, des implications bien au-delà du goût}

\section{Un rôle métabolique insoupçonné}

Émeline L. Maillet

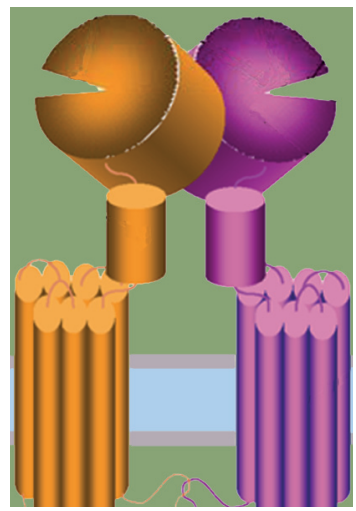

New York University

School of Medicine,

Department of Pharmacology, MSB, 550 First Avenue, New York, Ny 10016, États-Unis.

emeline.maillet@nyumc.org

dont les sucres, des petites molécules de diverses classes chimiques (saccharine, stevia ${ }^{2}$, acide glycyrrhizique ${ }^{3}$, cyclamate), des peptides (aspartame, D-tryptophane) et même des protéines (brazzéine,

Les édulcorants artificiels sont utilisés depuis de nombreuses années pour procurer une sensation sucrée sans apport calorique. Cependant, il a été récemment découvert que les récepteurs chémosenseurs des sucrants ne sont pas dévolus à la seule fonction du goût ni leur expression strictement restreinte aux cellules des papilles gustatives situées à la surface de la langue et du palais mou. Ils sont en fait exprimés en dehors du système gustatif, notamment dans des cellules chémosensorielles de l'intestin où ils interviendraient dans les systèmes endocriniens du tractus gastro-intestinal [6], mais aussi dans les reins, le foie, les testicules, le pancréas exocrine, certains lymphocytes, et même certaines régions du cerveau [7, 8] (Figure 2). Leurs rôles physiologiques dans la signalisation en réponse aux nutriments

\footnotetext{
${ }^{2}$ La stevia est un petit arbuste d'Amérique du Sud connu depuis des siècles par les Indiens d'Amazonie pour son pouvoir sucrant.

${ }^{3}$ L'acide glycyrrhizique est le principe actif de la réglisse, et son pouvoir sucrant est de 30 à 50 fois supérieur à celui du saccharose.

${ }^{4}$ Toutes trois sont des extraits de plantes cultivées en Afrique et ayant un fort pouvoir sucrant.
} 


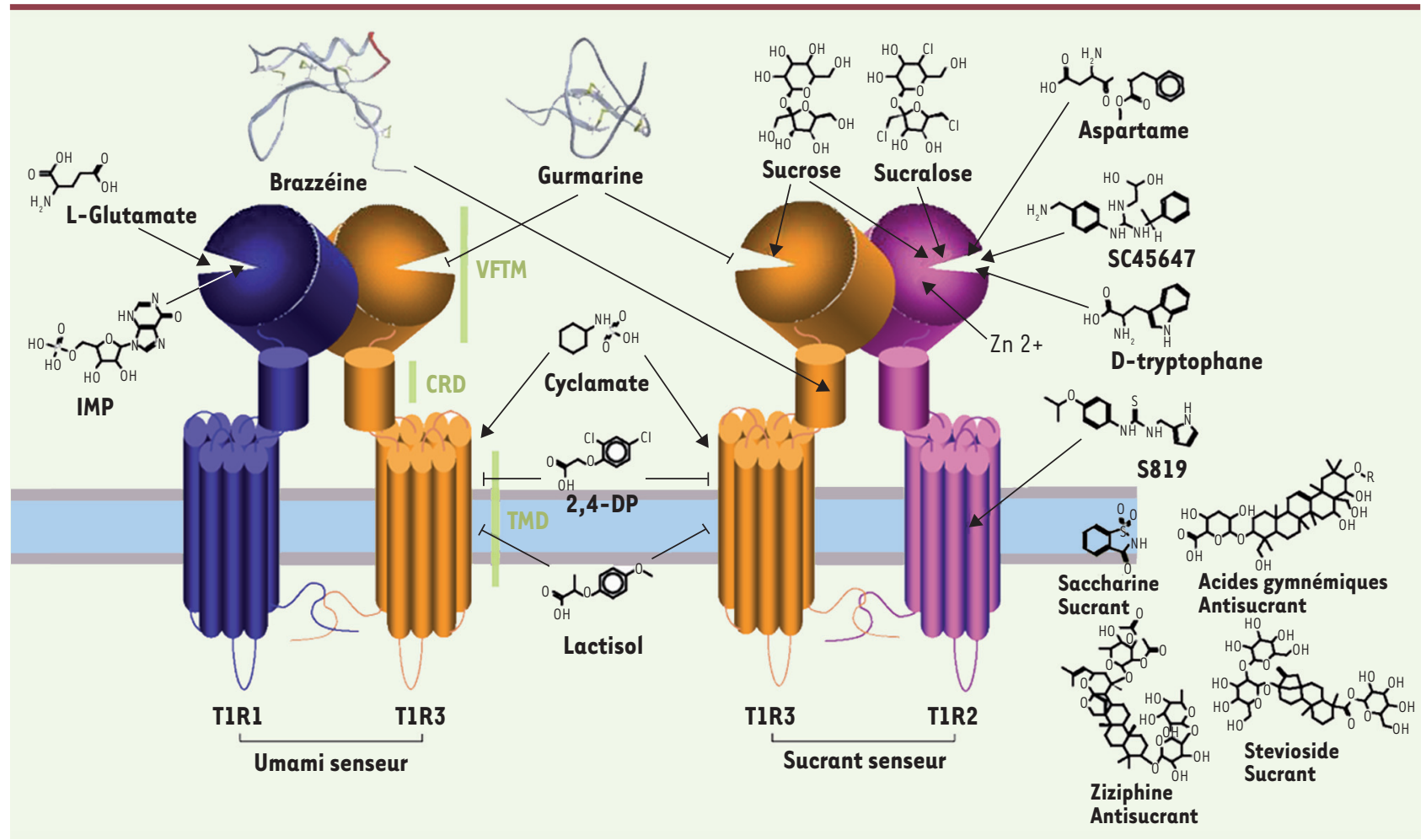

Figure 1. Récepteurs membranaires TIR aux sucrants et umamisants. TIR3 est la sous-unité commune aux récepteurs TIR2-TIR3 des composés sucrants et TIRI-TIR3 des composés umamisants. Les TIR présentent une large structure extracellulaire contenant le VFTM et le CRD et un motif transmembranaire (7TM) caractéristique des RCPG. Chacun de ces domaines est la cible de ligands agonistes très diversifiés allant des sucres (sucrose), acides aminés ( $D$-tryptophane, L-glutamate), dipeptides (aspartame) jusqu'à des protéines (monelline), mais aussi des petites molécules (saccharine, cyclamate, stéviosides) et certains ions (plomb, zinc). Flèches et flèches barrées indiquent respectivement une action agoniste et une action inhibitrice.

et le concept de chémosensibilité à l'extérieur du système gustatif commencent à susciter un intérêt notable.

En effet, des études récentes ont démontré que non seulement les sucres, mais aussi les édulcorants artificiels, sont des agonistes puissants des récepteurs $T 1 R$, que ce soit au niveau lingual ou au niveau intestinal $[6,9]$. Si les chémorécepteurs TIR des cellules gustatives signalent la présence d'aliments riches en glucides et en acides aminés au cerveau, ces mêmes récepteurs sont, par exemple, impliqués dans la régulation de la sécrétion d'incrétines (GLP- $1^{5}$ [glucagon-like peptide-1] et GIP [glucose-dependent insulinotropic polypeptide] dans les cellules intestinales de type L [6]. Le récepteur TIR3 serait le senseur de glucose au niveau de la lumière intestinale: celui-ci est impliqué dans l'induction du transport de glucose de type SGLT1 (sodium/glucose co-transporter 1 ), conduisant à une absorption accrue de glucides en réponse à une administration régulière de sucres mais aussi de l'édulcorant sucralose [10]. Finalement, il semble que

${ }^{5}$ Le GLP-1 est un polypeptide à effet anorexigène constitué de 30 acides aminés, sécrété par le tractus digestif, principalement l'iléon et le colon, sécrétion stimulée par la prise alimentaire. Le principal effet du GLP-1 est de stimuler la sécrétion d'insuline et de réduire la sécrétion de glucagon. II inhibe par ailleurs la sécrétion et la motilité gastriques et, par action hypothalamique, il réduit l'appétit et la prise alimentaire. le récepteur TIR3 soit directement engagé dans les mécanismes de sécrétion d'insuline au niveau des cellules pancréatiques, en réponse à des concentrations fortes de sucrose (Krokravlsiki et al., communication personnelle) et que les souris invalidées pour TIR3 aient une prédisposition accrue à des anomalies de leur régulation glycémique proches de celles d'en état diabétique [11]. De manière plus générale, une étude clinique [12] a récemment révélé une observation plutôt inattendue : l'ingestion de soda de type diet soda provoquerait un risque accru de syndrome métabolique (MetSyn) [13], dont on connaît les risques, cardiovasculaires notamment.

Finalement, ces études indiquent que les récepteurs chémosenseurs des molécules dites sapides, ici les récepteurs aux sucrants, et les protéines de signalisation qui leur sont associées, auraient un rôle important dans l'homéostasie du glucose et le métabolisme énergétique chez les mammifères. II semble donc pertinent d'émettre l'hypothèse selon laquelle une perturbation de leur activité pourrait influer, positivement ou 


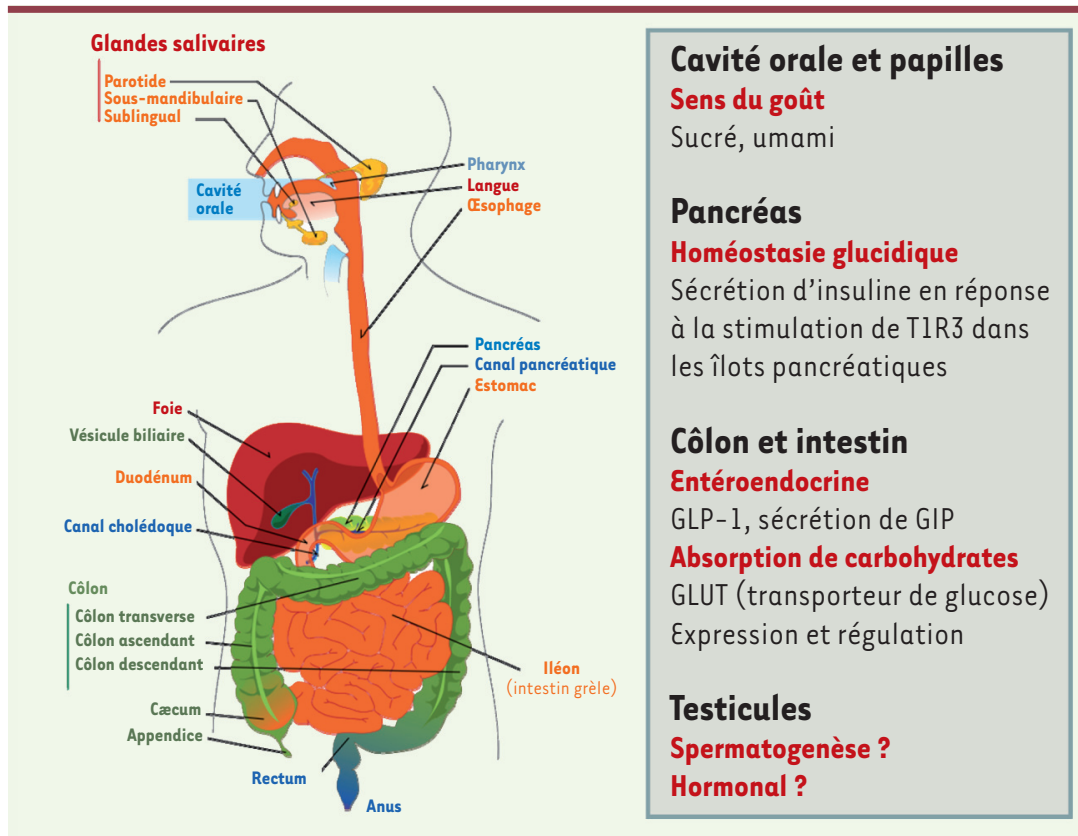

Figure 2. Expression des récepteurs chémosenseurs TIR. Les récepteurs TIR sont connus pour être les récepteurs du goût sucré et du goût umami. Cependant, ces récepteurs sont exprimés dans d'autres tissus et organes que les papilles de la langue et sembleraient participer à des fonctions physiologiques importantes telles que la sécrétion d'incrétines et d'insuline au niveau de l'intestin et du pancréas respectivement. L'étendue de leurs rôles et fonctions dans l'organisme et la raison de leur présence au niveau des testicules et $d u$ cerveau, par exemple, sont inconnues à ce jour (reproduit de Wikimedia commons, (C) dessin de Marina Ruiz).

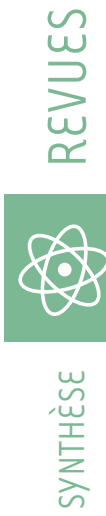

négativement selon la classe de composés modulateurs, sur l'évolution, voire la genèse de maladies comme le diabète de type II et l'obésité. Ces études sont encore préliminaires, mais les résultats obtenus jusqu'à présent ébranlent certaines idées (parfois élevées au rang de dogme) concernant des aliments dits de régime et a priori bénéfiques, contenant des édulcorants artificiels non caloriques comme substituts hédoniques aux sucres.

\section{Les molécules antisucrantes, une classe de composés méconnue}

Pendant les trente dernières années, beaucoup d'efforts ont porté sur la diversification des édulcorants de synthèse et l'accroissement de leur pouvoir agoniste, ainsi que sur le développement de molécules induisant des profils synergiques positifs, destinés à réduire la charge calorique en sucres dans l'alimentation humaine [14, 15]. On a aussi cherché des inhibiteurs des substances amères, notamment pour adoucir le goût de certaines préparations médicamenteuses. Toutefois, peu d'efforts de recherche ont été dédiés aux inhibiteurs des sensations sucrantes et umamisantes, probablement parce que les récepteurs du goût étaient considérés comme importants seulement pour la sensation du goût. Compte tenu de ce que l'on commence à savoir du rôle des récepteurs chémosensoriels en dehors du système gustatif, notamment de leurs implications métaboliques (Figure 2), le développement de molécules inhibitrices et/ou agissant en synergie négative pourrait représenter une approche novatrice en recherche pharmacologique et potentiellement, à plus long terme, pour la mise au point d'une nouvelle classe de médicaments.

\section{L'histoire montre l'exemple}

Ces molécules ne sont pas inconnues: ainsi, au cours de l'histoire humaine, des extraits et décoctions de plantes produisant naturelle- ment des substances inhibitrices du goût du sucre ont été spécifiquement utilisés pour leurs caractéristiques antisucrantes dans le traitement de certaines maladies. Depuis plus de 2000 ans en Inde, le Gymnema sylvestre, une plante tropicale qui contient des molécules antisucrantes de type acide gymnémique, a été utilisée pour traiter les patients atteints d' «urine sucrée » (diabétiques), combattre l'apport calorique de l'alimentation en cas de surpoids et réduire les fringales [16]. Les acides gymnémiques seraient des ligands antagonistes purs (sans effet agoniste inverse, voir ci-dessous) du récepteur humain $\mathrm{TlR} 2 / 3$, mais leur site de liaison précis sur le récepteur n'est pas encore pharmacologiquement caractérisé. Ils sont inactifs vis-à-vis du récepteur aux umamisants TlRl/3 [17, 18]. Au vu de la grande diversité chimique des composés capables d'activer le chémosenseur TIR2/3 (Figure 1), il est raisonnable d'espérer une diversité similaire, au moins en théorie, des molécules antisucrantes inhibitrices de ce même récepteur.

\section{Exemples d'inhibiteurs pharmacologiques de T1R3}

Le premier inhibiteur du récepteur TIR3 caractérisé sur un plan pharmacologique est le lactisole, acide méthoxy-phénoxy-propionique [3, 19]. C'est une substance qui peut être extraite à partir de fèves de café, de chiralité $S$ par source naturelle. Cet énantiomère est la forme active inhibitrice du récepteur (Figures $1 \mathrm{~A}$ et 3 ). Le site de liaison du lactisole a été localisé au niveau du domaine transmembranaire de la version humaine du récepteur TIR3 [19]. Le site de liaison à TIR3 diffère dans les autres espèces, ce qui explique que la 


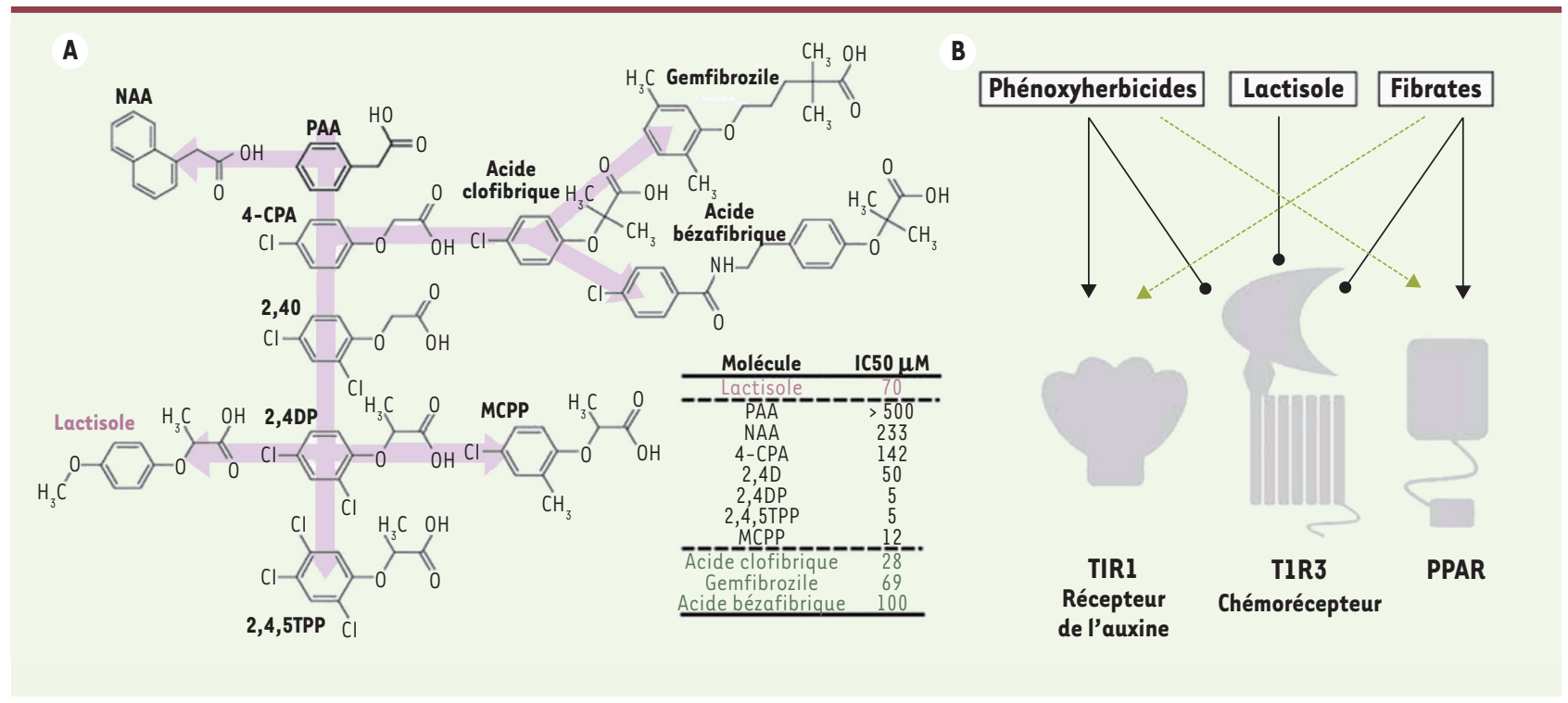

Figure 3. Similitude structurelle et fonctionnelle entre lactisole, phénoxyherbicides et fibrates. A. Le récepteur TIR3 est inhibé par le sucrant inverse lactisole, certains herbicides de type phénoxy-auxinique, et des médicaments de type fibrates tels que le clofibrate. $\boldsymbol{B}$. II existe un recouvrement d'activité entre ces composés au niveau de leurs fonctions auxinique et antilipidémiante, respectivement.

caractéristique antisucrante du lactisole soit spécifique à l'homme et aux grands singes. Le lactisole est un inhibiteur de l'ensemble des sucrants naturels et des édulcorants. Ce composé est néanmoins capable de produire une perception d'eau sucrée lorsqu'il est éliminé de la cavité buccale par de l'eau. Ces réponses post-application (arrière-goût) sont généralement observées lorsque l'activité basale du récepteur est restaurée à la suite de la dissociation d'un ligand de type agoniste inverse [20]. Ainsi, le lactisole serait un sucrant inverse plutôt qu'un antisucrant strict.

L'acétate de plomb, $\mathrm{Pb}^{3+}$, a un goût sucré prononcé (defrutum, sirop de sapa). Par ailleurs, l'ion zinc, $\mathrm{Zn}^{2+}$, est un inhibiteur de la saveur sucrée [14]. L'ion zinc est un ligand direct du récepteur T1R2/3 et son site de liaison est probablement localisé dans la partie extracellulaire du récepteur TIR2/3, au niveau d'une poche proximale au site de liaison des ligands agonistes de type orthostérique (Maillet et al., données non publiées).

D'une classe chimique très différente, la gurmarine, (en référence à gurmar qui signifie destructeur de sucre en hindi) est un polypeptide de 35 acides aminés extrait de feuilles de Gymnema sylvestre (mentionné plus haut) [21] (Figure 1). Cette substance supprime, chez les rongeurs, les mécanismes comportementaux de préférence et les réponses gustatives neuronales des rongeurs vis-à-vis des composés sucrés et umamisants, sans affecter les réponses aux saveurs salées, acides ou amères. La gurmarine n'a pas d'effet notable au niveau gustatif chez l'homme. Des études récentes montrent que cette petite protéine extrêmement stable (au motif cystéine-knot) est un ligand direct du récepteur TlR3; elle se lie à et dans la partie extracellulaire dite Venus flytrap module du récepteur pour bloquer son activation (Figure 1) [22]. Des études cherchant à définir les bases moléculaires de la spécificité interespèce de la gurmarine et à élucider le mécanisme précis d'interaction de cet antagoniste de haute affinité avec son récepteur sont en cours. Elles fourniront les bases nécessaires pour le développement de ligands dérivés, qui seraient potentiellement efficaces sur le récepteur de l'espèce humaine et destinés à la recherche médicale.

\section{Herbicides et fibrates : quel lien avec la chémosensation?}

Il existe des similitudes structurelles entre deux classes de composés: certains médicaments fibrates de première génération et certains herbicides auxiniques de type phénoxy-.

Les fibrates de première génération (acide clofibrique, bézafibrate) sont une classe de molécules thérapeutiques utilisées dans le traitement de nombreuses formes d'hyperlipidémie (Figure 3). Les récepteurs nucléaires de type peroxisome proliferator activated receptor, notamment alpha (PPAR- $\alpha$ ) [23], sont la cible thérapeutique des fibrates. Des études avec des agonistes PPAR ont également signalé une baisse du glucose circulant, une meilleure tolérance au glucose et une amélioration de la sensibilité à l'insuline [24], bien que le mécanisme d'action des fibrates sur l'homéostasie du glucose reste flou. Les phénoxyherbicides (2,4D; 2,4DP; MCPP ; 2,4,5T, Figure 3) sont une classe de composés organo-auxiniques largement utilisés en agriculture pour contrôler les mauvaises herbes à large feuillage [25]. Environ 25000 tonnes (au moins) de phénoxyherbicides sont 
utilisés chaque année aux États-Unis, et le composé 2,4D représente $86 \%$ de la consommation totale [26]. L'Environmental protection agency (EPA) a défini la toxicité aiguë par voie orale du 2,4DP comme étant légère, en se basant sur une DL50-rat ${ }^{6}$ de $537 \mathrm{mg} / \mathrm{kg}$. Cependant, l'absorption par le sol des phénoxyherbicides est faible, leur taux de lixiviation ${ }^{7}$ élevé, et leur potentiel de contamination des aliments et des nappes phréatiques existe.

Les fibrates et phénoxyherbicides semblent structurellement et, dans une certaine mesure, fonctionnellement, similaires. Par exemple, l'acide clofibrique (acide 2-[4-chlorophénoxy]-2-méthylpropanoïque), qui fait partie de la première génération de fibrates largement utilisée, a effectivement démontré une activité herbicide [27]. Réciproquement, certains phénoxyherbicides comme le 2,4D (acide 2,4-dichlorophénoxyacétique) et le MCPA (acide [4-chloro2-méthylphénoxy]acétique) ont des effets antilipidémiants semblables, chez les rats, à ceux qu'entraîne un fibrate [28].

Nous avons pu montrer dans un travail récent que certains phénoxyherbicides et certains fibrates, en raison de leur similitude structurelle avec le lactisole, sont de robustes inhibiteurs des récepteurs T1R2/3 in vitro [29] (Figure 3). Ces composés sont capables d'inhiber, dans des cellules HEK (human embryonic kidney), l'activation des récepteurs T1R2/3 induite par le sucrant sucralose et par d'autres édulcorants et sucrants naturels. Pour visualiser cette inhibition, les cellules sont transfectées par une construction qui leur fait exprimer une protéine $\mathrm{G}$ chimérique, G16-gus44, composée de la protéine $\mathrm{G} \alpha 16$, ligand des RCPG, et des 44 acides aminés carboxy-terminaux de la gusducine, qui interagit avec les récepteurs TIR. Lors de l'activation de la voie de transduction par G16-gus44, une réponse calcique est induite, qui peut être détectée [29]. II est donc possible de mesurer la réponse calcique lors de l'application de ces composés, phénoxyherbicides et fibrates, qui interagissent avec la partie transmembranaire de TIR3. Un simple test gustatif in vivo a montré en outre que le médicament clofibrate a effectivement la capacité de bloquer la sensation du goût sucré (TlR2/3), mais aussi du goût umami (TlRl/3), comme on pouvait s'y attendre pour un ligand de TlR3. Comme nous l'avons décrit plus haut pour le lactisole, le clofibrate a aussi la propriété d'induire un arrière-goût doux, clairement sucré, après rinçage de la bouche à l'eau après son administration. Ainsi, ces herbicides et fibrates seraient donc, en toute rigueur, des sucrants inverses (agonistes inverses) plutôt que des antisucrants (antagonistes).

Les médicaments fibrates testés dans cette étude (clofibrate, bezafibrate) interagissent avec le récepteur cible PPAR- $\alpha$ [24] avec une affinité comparable (environ 50 $\mathrm{MM}$ ) à celle déterminée pour leur interaction avec le récepteur TIR2/3 [29] (Figure 3A). Sur la base de cette équivalence d'efficacité, les niveaux intestinaux et plasmatiques d'acide clofibrique atteints au cours d'un traitement systémique par les fibrates seraient donc suffisants non seulement pour activer efficacement les récepteurs PPAR, mais aussi pour bloquer efficacement les récepteurs

${ }^{6}$ La dose létale 50 ou DL50 est un indicateur quantitatif de la toxicité d'une substance. C'est la masse de substance nécessaire pour tuer $50 \%$ des animaux dans un lot. Elle s'exprime en milligrammes de matière active par kilogramme d'animal. Plus ce chiffre est petit, plus la substance est toxique.

${ }^{7}$ La lixiviation désigne une technique de lessivage de produits solides par un solvant approprié.
TIR3. Les récepteurs TIR3 pourraient donc être une cible biologique primaire de certains fibrates chez l'homme et ainsi contribuer à certains de leurs effets sur le métabolisme des nutriments et de l'homéostasie du glucose (Figure 3B).

Quant aux phénoxyherbicides testés dans cette étude, ce sont des produits chimiques exclusivement dédiés à l'agriculture et au contrôle des mauvaises herbes. Cependant, certains d'entre eux ont une affinité pour le récepteur TIR3 humain supérieure à celle des fibrates, de l'ordre du $\mu \mathrm{M}$ (Figure $3 \mathrm{C}$ ). De plus, comme le lactisole, ces molécules sont actives sur la forme humaine du récepteur TIR3 alors qu'elles sont totalement inactives sur les récepteurs TIR de souris et de rats, animaux de laboratoire généralement utilisés pour les études biologiques et toxicologiques. II n'était donc pas pensable, jusqu'à ce jour, de pouvoir détecter des effets physiologiques sur les récepteurs cibles TIR dans les fonctions de nutrition et du métabolisme dans ces modèles animaux.

\section{Perspectives sur les antisucrants et les sucrants inverses}

Le lien entre phénoxyherbicides et fibrates a été souligné récemment dans une étude écologique et sanitaire réalisée dans les États producteurs de blé aux ÉtatsUnis : elle décrit une incidence en baisse pour certaines maladies cardiovasculaires et un effet bénéfique sur le métabolisme des lipides dans les populations exposées [30]. Mais une augmentation significative dans l'incidence de diabète de type II a été rapportée. Un tableau typique de diabète de type II ne se voit qu'après un long délai pendant lequel s'installe progressivement une dérégulation de l'homéostasie glucidique. On ne peut donc pas exclure des effets sur la santé humaine de certains composés herbicides contemporains massivement utilisés dont l'effet aurait été sous-estimé dans les études animales. En effet, on s'intéresse de plus en plus aux conséquences sanitaires possibles des produits chimiques perturbant le système endocrinien : ces substances qui sont présentes dans notre environnement, dans les aliments et les produits de grande consommation, interfèrent avec la biosynthèse des hormones, le métabolisme ou sa régulation, et entraînent une déviation du contrôle de l'homéostasie normale ou de la reproduction [31]. L'exposition modérée mais cependant prolongée ou répétée, à des composés actifs sur TIR3 pourrait, par exemple au niveau intestinal, avoir des conséquences sur les paramètres de réactivité et de temporalité qui contrôlent la libération d'hormones. Des dérives métaboliques à long terme pourraient être 
induites par le dysfonctionnement des récepteurs TlR. Des recherches plus nombreuses sur le sujet, utilisant des modèles animaux humanisés pour ces récepteurs, apporteront une meilleure information et répondront à ces préoccupations.

Les composés antisucrants, qui agissent puissamment et sélectivement sur les récepteurs TlR comme antagonistes purs ou agonistes inverses, commencent à être identifiés et seront développés. Ces composés fourniront à l'avenir les outils nécessaires pour la recherche fondamentale sur les rôles multiples, et qui restent à définir, des récepteurs chémosenseurs de la famille TIR (Figure 2). De plus, certains composés antisucrants et leurs dérivés pourraient offrir une nouvelle piste thérapeutique, potentiellement complémentaire et/ou synergique des approches et cibles actuelles. On peut peut-être anticiper leur intérêt dans le traitement du syndrome métabolique, de l'obésité ou encore du diabète de type II. $\diamond$

\section{SUMMARY}

\section{Modulation of TIR chemosensory receptors}

for sweet nutrients: new paradigms in metabolic regulation

Recent studies have demonstrated that the sweet-sensing receptors TlR2/3, thought to be "taste receptors" specifically expressed in lingual system, are also expressed and involved in the chemo-detection of sweetening molecules circulating in other organs. Researches that focus on their roles in intestinal absorption, metabolic regulation and glucose homeostasis, in particular, are increasing. Indeed, the sweet-sensing receptor could provide a new therapeutic target for certain metabolic disorders and diseases like obesity and diabetes. If the natural and artificial sweeteners agonists are diverse and well known, the "anti-sweeteners" antagonistic molecules are a class of compounds that received very little attention until now. Their potential roles and pharmacological relevance outside the taste system are discussed. Moreover, the recent finding that 2 major classes of compounds belonging respectively to the fields of medicine (fibrates) and agriculture (phenoxy-herbicides) are potent inhibitors of human TIR3 receptor is reported, raising new questions about their potential impact on human metabolism. $\diamond$

\section{REMERCIEMENTS}

L'auteur remercie le Dr Vincent Vialou pour sa lecture et ses commentaires sur le manuscrit.

\section{CONFLIT D'INTÉRÊTS}

L'auteur déclare n'avoir aucun conflit d'intérêts concernant les données publiées dans cet article.

\section{RÉFÉRENCES}

1. Chandrashekar J, Hoon MA, Ryba NJ, Zuker CS. The receptors and cells for mammalian taste. Nature $2006 ; 444: 288-94$.

2. Zhao GQ, Zhang $\mathrm{Y}$, Hoon MA, et al. The receptors for mammalian sweet and umami taste. Cell $2003 ; 115: 255-66$.

3. Xu H, Staszewski L, Tang H, et al. Different functional roles of TlR subunits in the heteromeric taste receptors. Proc Natl Acad Sci USA $2004 ; 101$ : 14258-63.

4. Pin JP, Galvez T, Prezeau L. Evolution, structure, and activation mechanism of family $3 / C$ G-protein-coupled receptors. Pharmacol Ther 2003 ; $98: 325-54$.
5. Cui M, Jiang P, Maillet $\varepsilon$, et al. The heterodimeric sweet taste receptor has multiple potential ligand binding sites. Curr Pharm Des $2006 ; 12: 4591-600$.

6. Jang HJ, Kokrashvili Z, Theodorakis MJ, et al. Gut-expressed gustducin and taste receptors regulate secretion of glucagon-like peptide-1. Proc Natl Acad Sci USA $2007 ; 104: 15069-74$.

7. Kiuchi S, Yamada T, Kiyokawa N, et al. Genomic structure of swine taste receptor family 1 member 3, TASIR3, and its expression in tissues. Cytogenet Genome Res 2006 ; $115: 51-61$.

8. Taniguchi K. Expression of the sweet receptor protein, $T 1 R 3$, in the human liver and pancreas. J Vet Med Sci $2004 ; 66$ : 1311-4.

9. Mace 0J, Affleck J, Patel N, Kellett GL. Sweet taste receptors in rat small intestine stimulate glucose absorption through apical GLUT2. J Physiol 2007 ; 582 : 379-92.

10. Margolskee RF, Dyer J, Kokrashvili Z, et al. TIR3 and gustducin in gut sense sugars to regulate expression of $\mathrm{Na+-glucose}$ cotransporter 1. Proc Natl Acad Sci USA $2007 ; 104: 15075-80$.

11. Egan JM, Margolskee RF. Taste cells of the gut and gastrointestinal chemosensation. Mol Interv $2008 ; 8: 78-81$.

12. Lutsey PL, Steffen LM, Stevens J. Dietary intake and the development of the metabolic syndrome: the atherosclerosis risk in communities study. Circulation $2008 ; 117: 754-61$.

13. Junquero D, Rival $Y$. Syndrome métabolique : quelle définition pour quel(s) traitement(s) ? Med Sci (Paris) $2005 ; 21: 1045-53$.

14. DuBois GE. Unraveling the biochemistry of sweet and umami tastes. Proc Natl Acad Sci USA 2004 ; 101 : 13972-3.

15. Tinti JM, Nofre C. Design of sweeteners. In : Walters DE, Orthoefer FT, DuBois GE, eds. Sweeteners: discovery, molecular design and chemoreception. Washington DC : Am Chem Soc, 1991, ser 450, 206-13.

16. Kanetkar P, Singhal R, Kamat M. Gymnema sylvestre: a memoir. J Clin Biochem Nutr $2007 ; 41: 77-81$.

17. Sanematsu K, Shigemura N, Jyotaki M, et al. Identification of the interaction site for gymnemic acid at the sweet taste receptor T1R2+T1R3. Chem Senses 2009; $34:$ A63-A.

18. Hellekant G, Ninomiya Y. On the taste of umami in chimpanzee. Physiol Behav $1991 ; 49: 927-34$.

19. Jiang $P$, Cui $M$, Zhao $B$, et al. Lactisole interacts with the transmembrane domains of human TIR3 to inhibit sweet taste. J Biol Chem $2005 ; 280$ : 15238-46.

20. Greasley PJ, Clapham JC. Inverse agonism or neutral antagonism at G-protein coupled receptors: a medicinal chemistry challenge worth pursuing? Eur Pharmacol 2006 ; 553 : 1-9.

21. Imoto T, Miyasaka A, Ishima R, Akasaka K. A novel peptide isolated from the leaves of Gymnema sylvestre. I. Characterization and its suppressive effect on the neural responses to sweet taste stimuli in the rat. Comp Biochem Physiol A Comp Physiol 1991; $100: 309-14$.

22. Maillet $\varepsilon L$, Pelletier L, Cardozo TJ, et al. Gurmarin inhibits the sweet receptor by binding to the venus fly trap module of TlR3. Chem senses 2009 ; 34 : A78-A.

23. Fruchart JC, Staels B, Duriez P. The role of fibric acids in atherosclerosis. Curr Atheroscler Rep $2001 ; 3: 83-92$.

24. Staels B, Fruchart JC. Therapeutic roles of peroxisome proliferator-activated receptor agonists. Diabetes $2005 ; 54: 2460-70$.

25. Kelley KB, Riechers DE. Recent developments in auxin biology and new opportunities for auxinic herbicide research. Pestic Biochem Physiol 2007 ; 89: 1-11.

26. Szmedra P. Banning 2,4-D and the phenoxy herbicides: potential economic impact. Weed Science $1997 ; 45: 592-8$.

27. Lahey KA, Yuan R, Burns JK, et al. Induction of phytohormones and differential gene expression in citrus flowers infected by the fungus Colletotrichum acutatum. Mol Plant Microbe Interact $2004 ; 17$ : 1394-401.

28. Vainio $H$, Linnainmaa $K$, Kahonen $M$, et al. Hypolipidemia and peroxisome proliferation induced by phenoxyacetic acid herbicides in rats. Biochem pharmacol $1983 ; 32: 2775-9$.

29. Maillet $\varepsilon L$, Margolskee RF, Mosinger B. Phenoxy herbicides and fibrates potently inhibit the human chemosensory receptor subunit TlR3. J Med Chem 2009; 52 : 6931-5.

30. Schreinemachers DM. Mortality from ischemic heart disease and diabetes mellitus (type 2) in four US wheat-producing states: a hypothesis-generating study. Environ Health Perspect 2006 ; 114 : 186-93.

31. Cravedi JP, Zalko D, Savouret JF, et al. Le concept de perturbation endocrinienne et la santé humaine. Med Sci (Paris) 2007 ; $23: 198-204$.

TIRÉS À PART

E.L. Maillet 